\title{
Heterosis Studies in Okra (Abelmoschus esculentus L. Moench)
}

\author{
Prakash Kerure $^{1^{*}}$ and M. Pitchaimuthu ${ }^{2}$ \\ ${ }^{1}$ ICAR-Krishi Vigyan Kendra, Chitradurga-577 598, Karnataka, India \\ ${ }^{2}$ ICAR-Indian Institute of Horticultural Research, Bengaluru-560 089, Karnataka, India
}

*Corresponding author

\begin{tabular}{|l|}
\hline Ke y w o r d s \\
$\begin{array}{l}\text { Abelmoschus esculentus, } \\
\text { Heterosis, Half diallel, } \\
\text { Fruit yield }\end{array}$ \\
\hline Article Info \\
\hline $\begin{array}{l}\text { Accepted: } \\
12 \text { August } 2018 \\
\text { Available Online: } \\
\text { 10 September } 2018\end{array}$ \\
\hline
\end{tabular}

\section{Introduction}

Okra (Abelmoschus esculentus (L.) Moench) an annual, often cross-pollinated crop belonging to the family Malvaceae, is an important crop of the tropics and subtropics of the world. The yield potential of okra is very low. However, it could be improved through hybridization. Marked heterosis of 38 to 71

\section{A B S T R A C T}

Heterosis is manifested through greater vigour of $F_{1}$ over their parents resulting into higher yields through their component characters and the study of heterosis would help in selection of heterotic crosses for commercial exploitation of $F_{1}$ hybrids in okra (Abelmoschus esculentus (L.) Moench). Forty five $\mathrm{F}_{1} \mathrm{~s}$ were developed by crossing 10 elite lines of okra in half diallel fashion during summer 2016. All $45 \mathrm{~F}_{1} \mathrm{~s}$ along with their 10 parents and one standard control (Nunhems hybrid Shakti) were evaluated in a randomized complete block design with three replicates during late kharif (July to October) 2016 at ICAR- Krishi Vigyan Kendra, Babbur Farm, Hiriyur, Chitradurga, Karnataka. The magnitude of heterosis varied from cross to cross for all the characters studied. The variance due to parents verses hybrids was highly significant $(\mathrm{P}=0.01)$ for plant height, number of branches per plant, first fruit producing node, fruit length, average fruit weight, number of fruits per plant and yield per plant. The results revealed wide range of heterotic pattern for different traits. For first fruit producing node, heterosis over better parent and standard control ranged from -25.44 to 54.32 and -9.03 to 43.32 respectively. For days to $50 \%$ flowering, heterosis over better parent and standard control ranged from -8.03 to 3.79 and -8.70 to 0.72 respectively and for total yield per plant, heterosis over better parent and standard control ranged from -32.66 to 83.78 and -0.13 to 168.55 respectively. Out of 45 $\mathrm{F}_{1} \mathrm{~s}, 44 \mathrm{~F}_{1} \mathrm{~s}$ crosses exhibit significant standard heterosis in any given direction for total yield per plant except cross IIHR-604 x IIHR-107 (-0.13\%). The maximum positive significant heterosis was observed in cross IIHR-875 x IIHR-478 (83.78\%) over better parent and (168.55\%) over standard parent may be exploited for commercial cultivation. 
offers quantum jump in yield in short period of time. Hybrid vigour in okra has been first reported by Vijayaraghavan and Warier (1946). For exploitation of heterosis, choice of suitable parents is an important pre-requisite. For exploitation of heterosis, choice of suitable parents is an important pre-requisite. Selection of parents on the basis of phenotypic performance alone is not a sound procedure. It is therefore essential that parents should be chosen on the basis of their combining ability. The half diallel mating design has been used in the present study to assess the genetic potentialities. The magnitude of heterosis provides a basis for genetical diversity and a guide for the choice of desirable parents for developing superior $F_{1}$ hybrids to exploit hybrid vigour and for building gene pools to be employed in future breeding programme. Keeping this in view, the present investigation was carried out to know magnitude of heterosis for fruit yield and its component traits in okra.

\section{Materials and Methods}

The experiment materials comprised of ten elite and nearly homozygous lines of okra namely IIHR -875, IIHR -478, IIHR- 604, IIHR- 567, IIHR- 182, IIHR- 595, IIHR- 562, IIHR- 347, IIHR- 444, IIHR-107 selected from the germplasm collected by ICAR-Indian Institute of Horticulture Research Institute, Bengaluru, Karnataka and were crossed in n(n - 1)/2 possible combinations during summer 2016 to generate the breeding material. The resulting 45 one way crosses along with their 10 counterpart parental lines and one standard control (Nunhems Hybrid Shakti) were evaluated in a randomized complete block design with three replicates. The experiment was conducted at the Experimental Farm, ICAR- Krishi Vigyan Kendra, Babbur Farm, Hiriyur, Chitradurga, Karnataka. The experiment was conducted during late kharif (July-October) 2016. The experimental site is situated in Central dry zone (Zone-4) $13^{\circ} 57^{\prime} 32^{\prime \prime}$ North latitude and 70'37'38' East longitude at 606 meters above Mean Sea Level (MSL). Among the agro-climatic zones of Karnataka, Hiriyur benefits of both SouthWest and North- East monsoons. The annual average rainfall for last 35 years is $567 \mathrm{~mm}$ per year. The maximum and minimum temperature in a year ranges between $31.49{ }^{\circ} \mathrm{C}$ and $20.91{ }^{\circ} \mathrm{C}$, respectively. The annual relative humidity of location is 77.76 per cent and 61.74 per cent in morning and evening, respectively. The soil type of the experimental block was sandy clay loamy (montmorillonite) with a uniform fertility having soil $\mathrm{pH}$ range of 7.0 to 8.0. The experimental plots were ploughed repeatedly and land was brought to a fine tilth. Raised beds with inline drip and plastic mulching developed at $90 \times 10 \times 30$ $\mathrm{cm}$. Two to three seeds of each genotype per hill were dibbled at a distance of $15 \mathrm{~cm}$ apart and $60 \mathrm{~cm}$ between rows on the beds.

Biometric data were recorded for 12 quantitative characters. Observations on the characters like plant height $(\mathrm{cm})$, number of branches per plant, internodal length $(\mathrm{cm})$, stem girth $(\mathrm{mm})$, first fruit producing node, fruit length $(\mathrm{cm})$, fruit diameter $(\mathrm{mm})$, number of ridges per fruit and average fruit weight $(\mathrm{g})$ were recorded on five randomly selected competitive plants, while the observations on the characters like days to $50 \%$ flowering, total number of fruits per plant and total yield per plant $(\mathrm{g})$ were recorded on whole plot basis in each entry in each replicate. Relative heterosis, heterobeltiosis and standard heterosis were determined as percent increase $(+)$ or decrease (-) of $F_{1}$ over mid parent (MP), better parent $(\mathrm{BP})$ and standard control (SC) using the formulae $\left(\mathrm{F}_{1}-\mathrm{MP} / \mathrm{MP} \times 100\right),\left(\mathrm{F}_{1^{-}}\right.$ $\mathrm{BP} / \mathrm{BP} \times 100)$ and $\left(\mathrm{F}_{1}-\mathrm{SC} / \mathrm{SC} \times 100\right)$, respectively (Singh, 1973). The statistical significance of heterosis, heterobeltiosis and standard heterosis was assessed by t-test (Wynne et al., 1970). 


\section{Results and Discussion}

The goal of okra hybrid breeding is to identify and then reliably reproduce superior hybrid genotypes. Virtually all commercial okra hybrids are made from crosses of inbred lines. Knowledge of heterotic groups from which to draw parental germplasm for hybrid combinations is limited.

Improvement of complex characters such as pod yield may be accomplished through the component approach of breeding. This method, in general, assumes strong associations of yield with a number of characters making up yield and simpler inheritance for these component characters.

\section{Analysis of variance}

\section{Genotypes (Treatments)}

ANOVA (Table 1) indicated significance of mean squares due to genotypes for almost all growth, earliness and yield parameters viz., plant height, intermodal length, number of branches per plant stem girth, first fruit producing node, days to $50 \%$ flowering, fruit length, fruit diameter, average fruit weight, number of ridges per fruit, number of fruits per plant and yield per plant.

This can be attributed to the fact that there were clear cut genotypic differences among the parents and their hybrids, which were phenotypically expressed.

\section{Parents}

Parents differed significantly, at $\mathrm{P}=0.01$ among themselves for all the growth, earliness and yield parameters, viz., plant height, internodal length, number of branches per plant stem girth, first fruit producing node, fruit length, fruit diameter, average fruit weight, number of ridges per fruit, number of fruits per plant and yield per plant. Whereas days to 50 per cent flowering, the variance due to parents was significant at $\mathrm{P}=0.05$.

\section{Hybrids (Crosses)}

There was highly significant differences $(\mathrm{P}=0.01)$ among the hybrids for all the growth, earliness and yield parameters studied.

\section{Parents vs. Hybrids}

The variance due to parents verses hybrids was highly significant $(\mathrm{P}=0.01)$ for plant height, number of branches per plant, first fruit producing node, fruit length, average fruit weight, number of fruits per plant and yield per plant. However, variance due to parent's verses hybrids was not significant for internodal length, stem girth, days to 50 per cent flowering, fruit diameter and number of ridges per fruit. Similar results were obtained by Mehta et al., 2007 and Hosamani et al., 2008.

\section{Heterosis}

The number of crosses in present study displaying significantly positive and negative heterosis over the better parent and standard control (Nunhems hybrid Shakti) are presented in Table 2. There was huge amount of variation in heterotic effects as they varied differently for different characters. For plant height, heterosis over better parent and standard control ranged from -33.30 to 13.93 and -23.82 to 30.12 per cent respectively. For this trait, nine cross over better parent and 36 cross over standard control manifested significantly positive heterosis. Heterosis over better parent and standard control ranged from -22.03 to 20.01 and -19.79 to 15.91 per cent respectively for internodal length. For internodal length 21 cross over better parent and 11 cross over standard control manifested significantly negative heterosis. 
For number of branches per plant, heterosis better parent and standard control ranged from -40.11 to 40.54 and -35.64 to 26.97 per cent respectively. For this trait, eight cross over better parent and four cross over standard control manifested significantly positive heterosis. For stem girth, heterosis over better parent and standard control ranged from 20.70 to 24.64 and -14.57 to 28.23 percent respectively. For this trait, seven cross over better parent and 18 cross over standard control manifested significantly positive heterosis.

For first fruit producing node, heterosis over better parent and standard control ranged from -25.44 to 54.32 and -9.03 to 43.32 percent respectively. For this trait, 15 cross over better parent and six cross over standard control manifested significant heterosis in desirable direction (negative). For days to $50 \%$ flowering, better parent and standard control ranged from -8.03 to 3.79 and -8.70 to 0.72 per cent respectively. For this trait, nine cross over better parent and nine cross over standard control manifested significantly negative heterosis.

For fruit length, better parent and standard control ranged from -25.40 to 24.30 and 25.24 to 13.53 respectively per cent (Table 3 ). For this trait, 10 cross over better parent and five cross over standard control manifested significantly positive heterosis. For fruit diameter, better parent and standard control ranged from -23.14 to 16.01 and -16.77 to 11.90 per cent respectively.

For this trait, seven cross over better parent and four cross over standard control manifested positively significant heterosis. For average fruit weight, better parent and standard control ranged from -30.05 to 31.76 and -15.39 to 42.25 respectively. For this trait, 16 cross over better parent and 26 cross over standard control manifested significantly positive heterosis. For number of ridges per fruit, better parent and standard control ranged from -13.07 to 15.09 and -3.85 to 17.31 per cent respectively. For this trait, two cross over better parent and eight cross over standard control manifested significantly positive heterosis.

For number of fruits per plant, heterosis over better parent and standard control ranged from -24.14 to 48.91 and -4.35 to 98.55 per cent respectively. For this trait, 19 cross over better parent and 42 cross over standard control manifested significantly positive heterosis. For total yield per plant, better parent and standard control ranged from -32.66 to 83.78 and -0.13 to 168.55 respectively per cent. For this trait, 20 cross over better parent and 44 cross over standard control manifested positively significant heterosis. From the results of the heterosis studies, it is evident that none of the $45 \mathrm{~F}_{1}$ hybrids of okra showed consistency in direction and degree of heterosis over three bases for all the characters studied. Some of them manifested positive heterosis while others exhibited negative heterosis, mainly due to varying extent of genetic diversity between parents of different cross combinations for the component characters. Significant heterosis was observed for all the growth, earliness and yield attributes. It is inferred that the magnitude of economic heterosis was higher for most of the growth and earliness characters under study.

In the present study, the estimates of relative heterosis, heterobeltiosis, and standard heterosis were found to be highly variable in direction and magnitude among crosses for all the characters under study. Weerasekara et al., (2007) and Jindal et al., (2009) also reported such a variation in heterosis for different characters. The manifestation of negative heterosis observed in some of the crosses for different traits may be due to the combination of the unfavorable genes of the parents. 
Fig.1 Per cent standard heterosis of top three hybrids IIHR-875 x IIHR-478, IIHR-604 x IIHR-347 and IIHR-478 x IIHR-567 for 12 characters studied

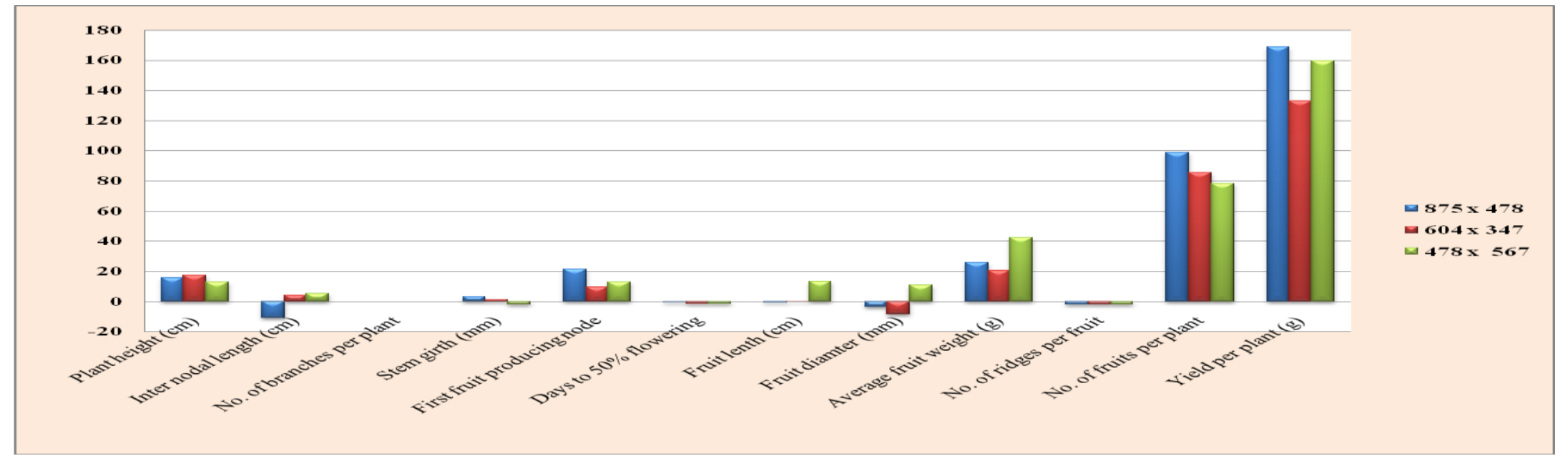

Table.1 Analysis of variance (mean sum of square) of combining abilities for twelve characters in a set of $10 \mathrm{X} 10$ half diallel crosses

\begin{tabular}{|c|c|c|c|c|c|c|c|c|c|c|c|c|c|}
\hline Source & d.f. & $\begin{array}{c}\text { Plant } \\
\text { height } \\
\text { (cm) }\end{array}$ & $\begin{array}{c}\text { Inter } \\
\text { nodal } \\
\text { length } \\
(\mathrm{cm})\end{array}$ & $\begin{array}{c}\text { No. of } \\
\text { branches } \\
\text { per plant }\end{array}$ & $\begin{array}{l}\text { Stem } \\
\text { girth } \\
(\mathrm{mm})\end{array}$ & $\begin{array}{c}\text { First } \\
\text { fruit } \\
\text { producin } \\
\text { g node }\end{array}$ & $\begin{array}{c}\text { Days to } \\
\mathbf{5 0 \%} \\
\text { flowering }\end{array}$ & $\begin{array}{c}\text { Fruit } \\
\text { length } \\
\text { (cm) }\end{array}$ & $\begin{array}{l}\text { Fruit } \\
\text { diamete } \\
\text { r (mm) }\end{array}$ & $\begin{array}{c}\text { Average } \\
\text { fruit } \\
\text { weight (g) }\end{array}$ & $\begin{array}{l}\text { No. of } \\
\text { ridges } \\
\text { per fruit }\end{array}$ & $\begin{array}{c}\text { No. of } \\
\text { fruits per } \\
\text { plant }\end{array}$ & $\begin{array}{l}\text { Yield per } \\
\text { plant } \\
\text { (g) }\end{array}$ \\
\hline Replicates & 2 & 0.39 & $1.78 * *$ & $1.42 * *$ & 0.65 & 0.01 & $37.98 * *$ & 0.34 & $2.23 * *$ & 0.84 & $0.57 * *$ & $49.32 * *$ & $9275.55 * *$ \\
\hline Treatments & 54 & $727.47 * *$ & $3.24 * *$ & $0.87 * *$ & $10.71 * *$ & $1.56 * *$ & $3.93 * *$ & $5.01 * *$ & $4.46 * *$ & $14.27 * *$ & $0.15 * *$ & $79.30 * *$ & $38483.22 * *$ \\
\hline Parents & 9 & $822.46 * *$ & $4.50 * *$ & $1.04 * *$ & $8.79 * *$ & $2.32 * *$ & $2.01 *$ & $3.34 * *$ & $4.22 * *$ & $11.07 * *$ & $0.16 * *$ & $13.31 * *$ & $15676.11 * * *$ \\
\hline Hybrids & 44 & $717.18 * *$ & $3.05 * *$ & $0.84 * *$ & $11.33 * *$ & $1.41 * *$ & $4.41 * *$ & $5.35 * *$ & $4.61 * *$ & $14.60 * *$ & $0.15 * *$ & $88.78 * *$ & $40219.54 * *$ \\
\hline $\begin{array}{c}\text { Parent Vs. } \\
\text { Hybrids }\end{array}$ & 1 & $324.95 * *$ & 0.01 & $0.77 * *$ & 0.51 & $1.72 * *$ & 0.10 & $5.17 * *$ & 0.01 & $28.71 * *$ & 0.00 & $256.02 * *$ & $167349.10 * *$ \\
\hline Error & 108 & 1.27 & 0.35 & 0.05 & 0.36 & 0.04 & 0.94 & 0.26 & 0.27 & 0.29 & 0.01 & 0.85 & 158.41 \\
\hline
\end{tabular}

$*$, **Significant at $\mathrm{P} \leq 0.05$ and $\mathrm{P} \leq 0.01$ levels probability, respectively. 
Table.2 Estimation of better parent and standard heterosis of 10 X 10 half diallel crosses in okra for different characters

\begin{tabular}{|c|c|c|c|c|c|c|c|c|c|c|c|c|c|}
\hline \multirow[t]{2}{*}{ Sl. No. } & \multirow[t]{2}{*}{ Crosses } & \multicolumn{2}{|c|}{ Plant height (cm) } & \multicolumn{2}{|c|}{ Inter nodal length (cm) } & \multicolumn{2}{|c|}{$\begin{array}{c}\text { No. of branches per } \\
\text { plant }\end{array}$} & \multicolumn{2}{|c|}{ Stem girth (mm) } & \multicolumn{2}{|c|}{$\begin{array}{l}\text { First fruit producing } \\
\text { node }\end{array}$} & \multicolumn{2}{|c|}{$\begin{array}{l}\text { Days to } \mathbf{5 0 \%} \\
\text { flowering }\end{array}$} \\
\hline & & BP & SC & BP & SC & BP & SC & BP & SC & $\mathbf{B P}$ & SC & $\mathbf{B P}$ & SC \\
\hline 1 & $875 \times 478$ & 0.05 & $15.83 * *$ & 9.08 & $-10.55 *$ & $32.58 * *$ & $20.06 * *$ & $-11.09 * *$ & 3.16 & $30.65 * *$ & $21.33 * *$ & 1.48 & -0.72 \\
\hline 2 & $875 \times 604$ & $13.93 * *$ & $30.12 * *$ & $-16.15 * *$ & $-14.72 * *$ & $33.98 * *$ & $21.32 * *$ & 2.77 & $10.71 * *$ & $54.32 * *$ & $43.32 * *$ & 0.00 & -0.72 \\
\hline 3 & $875 \times 567$ & $-2.12 * *$ & $28.81 * *$ & $20.01 * *$ & $15.91 * *$ & $13.66 *$ & 2.92 & $4.67 *$ & $10.78 * *$ & -3.48 & $17.05 * *$ & -0.73 & -1.45 \\
\hline 4 & $875 \times 182$ & $6.27 * *$ & $24.27 * *$ & -4.31 & -1.48 & 2.89 & -2.82 & -0.23 & 1.49 & -0.05 & $13.07 * *$ & 1.49 & -1.45 \\
\hline 5 & $875 \times 595$ & $3.79 * *$ & $27.20 * *$ & $-17.46 * *$ & $-11.10 * *$ & 0.32 & -9.15 & 2.94 & $4.72 *$ & 4.08 & $13.78 * *$ & $3.79 *$ & -0.72 \\
\hline 6 & $875 \times 562$ & $3.49 * *$ & $23.45 * *$ & 7.32 & $10.40 *$ & $31.51 * *$ & $19.08 * *$ & 0.39 & $5.45 *$ & 2.62 & $27.99 * *$ & -0.73 & -1.45 \\
\hline 7 & $875 \times 347$ & $5.02 * *$ & $25.72 * *$ & $-10.09 *$ & -4.46 & $-26.19 * *$ & -9.15 & 2.83 & $5.79 *$ & $23.56 * *$ & $38.03 * *$ & 1.49 & -1.45 \\
\hline 8 & $875 \times 444$ & $-4.38 * *$ & $8.34 * *$ & 9.13 & -6.49 & -12.04 & $-20.35 * *$ & -1.54 & 3.50 & $17.47 * *$ & $9.09 * *$ & $-5.15 * *$ & $-6.52 * *$ \\
\hline 9 & $875 \times 107$ & $-1.42 *$ & $11.69 * *$ & 7.19 & $14.00 * *$ & -5.70 & $-14.61 *$ & 0.73 & 2.48 & $-8.66 * *$ & -4.16 & $3.79 *$ & -0.72 \\
\hline 10 & $478 \times 604$ & $6.31 * *$ & $23.08 * *$ & 2.19 & 3.94 & $-25.93 * *$ & $-33.79 * *$ & -1.08 & $14.78 * *$ & 4.08 & -4.58 & -1.46 & -2.17 \\
\hline 11 & $478 \times 567$ & $-14.21 * *$ & $12.90 * *$ & $9.00 *$ & 5.27 & 2.29 & -8.57 & $-15.60 * *$ & -2.07 & $-6.76 * *$ & $13.07 * *$ & -0.73 & -1.45 \\
\hline 12 & $478 \times 182$ & $2.84 * *$ & $20.26 * *$ & $-10.98 * *$ & -8.35 & 4.54 & -1.27 & $-8.97 * *$ & $5.63 *$ & -4.94 & $7.55 *$ & 0.74 & -1.45 \\
\hline 13 & $478 \times 595$ & $-5.45 * *$ & $15.88 * *$ & 5.84 & $14.00 * *$ & $-21.24 * *$ & $-29.60 * *$ & $-14.34 * *$ & -0.60 & $-10.33 * *$ & -1.96 & 2.22 & 0.00 \\
\hline 14 & $478 \times 562$ & -0.11 & $19.15 * *$ & 4.73 & 7.74 & 2.29 & -8.57 & -0.66 & $15.27 * *$ & $-13.01 * *$ & $8.50 * *$ & -1.46 & -2.17 \\
\hline 15 & $478 \times 347$ & $2.18 * *$ & $22.32 * *$ & $8.04 *$ & $14.81 * *$ & $-40.11 * *$ & $-26.29 * *$ & $-15.19 * *$ & -1.59 & 3.67 & $15.81 * *$ & 0.00 & -2.17 \\
\hline 16 & $478 \times 444$ & $-14.13 * *$ & -0.58 & 0.68 & $-13.74 * *$ & $-18.63 * *$ & $-27.26 * *$ & $-11.15 * *$ & 3.10 & $17.81 * *$ & -4.10 & -0.74 & -2.17 \\
\hline 17 & $478 \times 107$ & $6.72 * *$ & $23.56 * *$ & -0.08 & 6.26 & -9.04 & $-18.70 * *$ & $-9.55 * *$ & $4.96 *$ & $-6.00 *$ & -1.37 & 1.48 & -0.72 \\
\hline 18 & $604 \times 567$ & $-13.23 * *$ & $14.19 * *$ & 2.22 & 3.97 & -12.42 & $-24.44 * *$ & $6.15 * *$ & $14.35 * *$ & $-10.14 * *$ & $8.97 * *$ & 0.73 & 0.00 \\
\hline 19 & $604 \times 182$ & $-5.82 * *$ & $10.13 * *$ & $-12.89 * *$ & $-10.32 *$ & -11.24 & $-16.16 * *$ & $-13.66 * *$ & $-6.99 * *$ & 2.00 & $15.39 * *$ & 0.00 & -0.72 \\
\hline 20 & $604 \times 595$ & $-23.61 * *$ & $-6.38 * *$ & -5.00 & 2.32 & -2.62 & $-20.25 * *$ & $-20.70 * *$ & $-14.57 * *$ & $-13.37 * *$ & -5.29 & $-5.11 * *$ & $-5.80 * *$ \\
\hline 21 & $604 \times 562$ & $-14.31 * *$ & $2.22 * *$ & -7.38 & -4.72 & -2.73 & $-20.35 * *$ & $-12.66 * *$ & $-5.91 *$ & $-9.24 * *$ & $13.19 * *$ & 0.73 & 0.00 \\
\hline 22 & $604 \times 347$ & $-2.01 * *$ & $17.30 * *$ & -1.91 & 4.23 & $-34.89 * *$ & $-19.86 * *$ & $-5.92 * *$ & 1.35 & -1.81 & $9.69 * *$ & -0.73 & -1.45 \\
\hline
\end{tabular}


Int.J.Curr.Microbiol.App.Sci (2018) 7(9): 1851-1862

\begin{tabular}{|c|c|c|c|c|c|c|c|c|c|c|c|c|c|}
\hline 23 & $604 \times 444$ & $-33.30 * *$ & $-23.82 * *$ & -1.20 & 0.49 & $-17.48 *$ & $-32.42 * *$ & -2.79 & $4.72 *$ & $6.93 *$ & -1.96 & -2.19 & -2.9 \\
\hline 24 & $604 \times 107$ & $-9.38 * *$ & $3.51 * *$ & $-10.74 * *$ & -5.07 & $-21.40 * *$ & $-35.64 * *$ & $-14.67 * *$ & $-8.07 * *$ & $-10.53 * *$ & -6.12 & 0.73 & 0.00 \\
\hline 25 & $567 \times 182$ & $-22.03 * *$ & $2.61 * *$ & $-14.58 * *$ & $-12.05 * *$ & -3.09 & -8.47 & $-5.35 *$ & 0.17 & $-9.90 * *$ & $9.27 * *$ & 0.00 & -0.72 \\
\hline 26 & $567 \times 595$ & $-5.15 * *$ & $24.82 * *$ & -5.22 & 2.09 & 2.26 & $-11.78 *$ & -0.99 & $4.78 *$ & $5.63 *$ & $28.10 * *$ & 1.46 & 0.72 \\
\hline 27 & $567 \times 562$ & $-12.11 * *$ & $15.67 * *$ & $-14.17 * *$ & $-11.71 * *$ & 8.35 & -6.52 & -4.14 & 1.45 & $-9.77 * *$ & $12.54 * *$ & $-5.11 * *$ & $-5.80 * *$ \\
\hline 28 & $567 \times 347$ & $-12.93 * *$ & $14.59 * *$ & $-14.45 * *$ & $-9.10 *$ & $-19.78 * *$ & -1.27 & 0.17 & $6.01 *$ & -3.87 & $16.58 * *$ & $-7.30 * *$ & $-7.97 * *$ \\
\hline 29 & $567 \times 444$ & $-22.81 * *$ & $1.58 *$ & -1.56 & -4.93 & -7.34 & $-20.06 * *$ & $7.73 * *$ & $14.02 * *$ & $-9.26 * *$ & $10.04 * *$ & $-7.30 * *$ & $-7.97 * *$ \\
\hline 30 & $567 \times 107$ & $-25.66 * *$ & $-2.16 * *$ & $-12.81 * *$ & -7.27 & $-16.14 *$ & $-27.65 * *$ & -0.86 & $4.93 *$ & $-24.99 * *$ & $-9.03 * *$ & $-8.03 * *$ & $-8.70 * *$ \\
\hline 31 & $182 \times 595$ & $-12.35 * *$ & $7.41 * *$ & -2.04 & 5.51 & $-20.82 * *$ & $-25.22 * *$ & $-10.19 * *$ & $-12.32 * *$ & $-19.01 * *$ & $-8.38 * *$ & 0.75 & -2.17 \\
\hline 32 & $182 \times 562$ & $-4.40 * *$ & $14.03 * *$ & -2.65 & 0.23 & $15.77 *$ & 9.35 & $-6.23 * *$ & -1.51 & $-10.29 * *$ & $11.88 * *$ & -2.19 & -2.90 \\
\hline 33 & $182 \times 347$ & $-11.24 * *$ & $6.25 * *$ & $-10.91 * *$ & -5.33 & 3.16 & $26.97 * *$ & 1.50 & 4.42 & 2.99 & $16.52 * *$ & 1.49 & -1.45 \\
\hline 34 & $182 \times 444$ & $-24.72 * *$ & $-11.98 * *$ & $-10.05 *$ & -7.39 & -8.25 & $-13.34 *$ & $-17.34 * *$ & $-13.11 * *$ & $-9.40 * *$ & 2.50 & -1.47 & -2.90 \\
\hline 35 & $182 \times 107$ & -0.90 & $15.88 * *$ & $-8.34 *$ & -2.52 & 3.40 & -2.34 & $9.79 * *$ & -0.68 & -1.79 & $11.11 * *$ & 1.49 & -1.45 \\
\hline 36 & $595 \times 562$ & $-6.46 * *$ & $14.64 * *$ & $-21.74 * *$ & $-15.71 * *$ & $35.57 * *$ & -1.66 & $8.61 * *$ & $14.08 * *$ & $-7.91 * *$ & $14.85 * *$ & 0.00 & -0.72 \\
\hline 37 & $595 \times 347$ & $-3.68 * *$ & $18.04 * *$ & $-8.10 *$ & -1.01 & $-10.60 *$ & 10.03 & $24.64 * *$ & $28.23 * *$ & 0.80 & $12.60 * *$ & $-5.97 * *$ & $-8.70 * *$ \\
\hline 38 & $595 \times 444$ & $-17.67 * *$ & 0.90 & $-14.10 * *$ & -7.48 & -4.37 & $-29.60 * *$ & $-12.53 * *$ & $-8.06 * *$ & $-12.34 * *$ & -4.16 & 2.21 & 0.72 \\
\hline 39 & $595 \times 107$ & 0.06 & $22.63 * *$ & -7.61 & -0.49 & $32.05 * *$ & $-13.34 *$ & $7.91 * *$ & $5.36 *$ & $-14.35 * *$ & $-6.36 *$ & 2.27 & -2.17 \\
\hline 40 & $562 \times 347$ & $-3.88 * *$ & $15.06 * *$ & -3.98 & 2.03 & $-24.84 * *$ & -7.50 & $-11.92 * *$ & $-7.49 * *$ & $-18.01 * *$ & 2.26 & 0.73 & 0.00 \\
\hline 41 & $562 \times 444$ & $-24.39 * *$ & $-9.81 * *$ & $-22.03 * *$ & $-19.79 * *$ & 13.36 & $-16.55 * *$ & $-18.20 * *$ & $-14.02 * *$ & $-25.44 * *$ & $-7.01 *$ & $-8.03 * *$ & $-8.70 * *$ \\
\hline 42 & $562 \times 107$ & $-6.17 * *$ & $11.92 * *$ & $-13.13 * *$ & -7.62 & $40.54 * *$ & 1.95 & $-4.69 *$ & 0.11 & $-11.62 * *$ & $10.22 * *$ & $-3.65 *$ & $-4.35 *$ \\
\hline 43 & $347 \times 444$ & $-19.22 * *$ & $-3.30 * *$ & $-9.19 *$ & -3.51 & $-17.17 * *$ & 1.95 & $-17.04 * *$ & $-12.79 * *$ & $-16.86 * *$ & $-7.13 *$ & -1.47 & -2.90 \\
\hline 44 & $347 \times 107$ & $-11.17 * *$ & $6.33 * *$ & $-11.44 * *$ & -5.82 & $-19.07 * *$ & -0.39 & $-11.97 * *$ & $-9.44 * *$ & $-9.57 * *$ & 1.01 & 1.49 & -1.45 \\
\hline 45 & $444 \times 107$ & $-15.35 * *$ & $-11.13 * *$ & $-20.08 * *$ & $-15.01 * *$ & -1.06 & $-27.17 * *$ & $-17.75 * *$ & $-13.54 * *$ & $-12.63 * *$ & $-8.32 * *$ & -1.47 & -2.90 \\
\hline & S.E.D & 0.92 & 0.92 & 0.48 & 0.48 & 0.20 & 0.20 & 0.49 & 0.49 & 0.17 & 0.17 & 0.79 & 0.79 \\
\hline & C.D. 0.05 & 1.86 & 1.86 & 0.97 & 0.97 & 0.39 & 0.39 & 0.99 & 0.99 & 0.35 & 0.35 & 1.60 & 1.60 \\
\hline & C.D. 0.01 & 2.42 & 2.42 & 1.28 & 1.28 & 0.51 & 0.51 & 1.29 & 1.29 & 0.45 & 0.45 & 2.08 & 2.08 \\
\hline
\end{tabular}


Table.3 Estimation of better parent and standard heterosis of 10 X 10 half diallel crosses in okra for different characters

\begin{tabular}{|c|c|c|c|c|c|c|c|c|c|c|c|c|c|}
\hline \multirow[t]{2}{*}{ SI. No. } & \multirow[t]{2}{*}{ Hybrids } & \multicolumn{2}{|c|}{ Fruit length (cm) } & \multicolumn{2}{|c|}{ Fruit diameter (mm) } & \multicolumn{2}{|c|}{ Average fruit weight (g) } & \multicolumn{2}{|c|}{ No. of ridges per fruit } & \multicolumn{2}{|c|}{$\begin{array}{c}\text { No. of fruits per } \\
\text { plant }\end{array}$} & \multicolumn{2}{|c|}{ Yield per plant (g) } \\
\hline & & BP & SC & $\mathbf{B P}$ & SC & BP & SC & BP & SC & BP & SC & BP & SC \\
\hline 1 & $875 \times 478$ & $8.53 * *$ & -0.88 & 0.02 & -3.52 & $20.55 * *$ & $25.88 * *$ & $-13.07 * *$ & -1.92 & $48.91 * *$ & $98.55 * *$ & $83.78 * *$ & $168.55 * *$ \\
\hline 2 & $875 \times 604$ & 3.52 & -0.79 & 3.79 & -3.21 & $29.58 * *$ & $16.02 * *$ & $-5.11 * *$ & $7.05 * *$ & $20.69 * *$ & $52.17 * *$ & $65.83 * *$ & $80.14 * *$ \\
\hline 3 & $875 \times 567$ & -0.13 & $-13.94 * *$ & $10.49 * *$ & $5.68 *$ & $6.40 *$ & $23.69 * *$ & $-12.50 * *$ & -1.28 & 3.33 & $34.78 * *$ & $9.57 * *$ & $73.80 * *$ \\
\hline 4 & $875 \times 182$ & -1.25 & $-14.90 * *$ & $5.06 *$ & $-4.73^{*}$ & -2.12 & 1.14 & $-3.98 *$ & $8.33 * *$ & $7.14 * *$ & $30.43 * *$ & 4.63 & $38.12 * *$ \\
\hline 5 & $875 \times 595$ & 2.85 & 0.83 & -3.60 & $6.19 * *$ & $8.71 * *$ & $20.18 * *$ & $-7.95 * *$ & $3.85 *$ & $10.34 * *$ & $39.13 * *$ & $28.82 * *$ & $82.04 * *$ \\
\hline 6 & 875 x 562 & $12.02 * *$ & -1.22 & $6.24 *$ & -4.39 & $30.22 * *$ & $16.68 * *$ & $3.41 *$ & $16.67 * *$ & 1.22 & $20.29 * *$ & $37.77 * *$ & $49.99 * *$ \\
\hline 7 & $875 \times 347$ & $-18.94 * *$ & $-17.60 * *$ & -0.30 & $-9.93 * *$ & $-20.04 * *$ & $-11.78 * *$ & $-13.07 * *$ & -1.92 & $46.74 * *$ & $95.65 * *$ & $15.87 * *$ & $77.61 * *$ \\
\hline 8 & $875 \times 444$ & -0.76 & $-14.48 * *$ & 1.79 & -3.16 & $-16.81 * *$ & 2.89 & $-10.23 * *$ & 1.28 & $11.90 * *$ & $36.23 * *$ & $-7.92 * *$ & $46.70 * *$ \\
\hline 9 & $875 \times 107$ & $20.58 * *$ & 5.27 & 2.02 & -2.87 & 2.28 & $8.14 * *$ & $-10.80 * *$ & $* 0.64$ & $26.92 * *$ & $43.48 * *$ & $37.79 * *$ & $61.55 * *$ \\
\hline 10 & $478 \times 604$ & 2.3 & -1.96 & $-13.72 * *$ & $-16.77 * *$ & $9.12 * *$ & $13.94 * *$ & -0.64 & -0.64 & 3.26 & $37.68 * *$ & $17.18 * *$ & $71.23 * *$ \\
\hline 11 & $478 \times 567$ & $24.30 * *$ & $13.53 * *$ & $15.00 * *$ & $10.94 * *$ & $22.37 * *$ & $42.25 * *$ & 0.00 & -1.92 & $33.70 * *$ & $78.26 * *$ & $63.61 * *$ & $159.53 * *$ \\
\hline 12 & $478 \times 182$ & $8.18 *$ & -1.19 & 0.11 & -3.44 & $22.68 * *$ & $28.11 * *$ & 0.00 & -1.28 & 1.09 & $34.78 * *$ & $25.54 * *$ & $83.45 * *$ \\
\hline 13 & $478 \times 595$ & 3.35 & 1.33 & $-16.13 * *$ & $-7.62 * *$ & $14.46 * *$ & $26.53 * *$ & 1.31 & -0.64 & 2.17 & $36.23 * *$ & $25.62 * *$ & $83.56 * *$ \\
\hline 14 & $478 \times 562$ & $6.83 *$ & -2.43 & -0.11 & -3.64 & 5.45 & $10.11 * *$ & 0.64 & $* 0.64$ & $30.43 * *$ & $73.91 * *$ & $33.87 * *$ & $95.62 * *$ \\
\hline 15 & $478 \times 347$ & $7.44 * *$ & $9.21 * *$ & -3.95 & $-7.35 * *$ & $10.73 * *$ & $22.18 * *$ & -2.58 & -3.21 & $-5.43 *$ & $26.09 * *$ & 3.58 & $58.77 * *$ \\
\hline 16 & $478 \times 444$ & -2.79 & $-11.21 * *$ & $16.01 * *$ & $11.90 * *$ & 1.10 & $25.04 * *$ & $15.09 * *$ & $17.31 * *$ & $-15.22 * *$ & $13.04 * *$ & $-5.37 * *$ & $50.76 * *$ \\
\hline 17 & $478 \times 107$ & $16.84 * *$ & $6.71 *$ & 0.62 & -2.94 & $13.54 * *$ & $20.05 * *$ & 1.30 & 0.00 & 0.00 & $33.33 * *$ & $19.20 * *$ & $74.18 * *$ \\
\hline 18 & $604 \times 567$ & -4.32 & $-8.31 * *$ & -3.13 & $-7.35 * *$ & -0.55 & $15.61 * *$ & -3.21 & -3.21 & 4.44 & $36.23 * *$ & 0.34 & $59.16 * *$ \\
\hline 19 & $604 \times 182$ & $10.71 * *$ & $6.10 *$ & $7.64 * *$ & 0.37 & $31.76 * *$ & $36.14 * *$ & -1.28 & -1.28 & $-13.79 * *$ & $8.70 * *$ & $20.02 * *$ & $58.43 * *$ \\
\hline 20 & $604 \times 595$ & $-10.52 * *$ & $-12.27 * *$ & $-23.14 * *$ & $-15.34 * *$ & 2.85 & $13.70 * *$ & -1.92 & -1.92 & $-6.90 * *$ & $17.39 * *$ & 1.79 & $43.83 * *$ \\
\hline 21 & $604 \times 562$ & 4.49 & 0.14 & 3.01 & -3.95 & $17.29 * *$ & 2.45 & 3.21 & 3.21 & 0.00 & $26.09 * *$ & $19.76 * *$ & $30.38 * *$ \\
\hline 22 & $604 \times 347$ & -1.84 & -0.23 & -1.64 & $-8.28 * *$ & $9.17 * *$ & $20.45 * *$ & -1.92 & -1.92 & $39.13 * *$ & $85.51 * *$ & $52.04 * *$ & $133.05 * *$ \\
\hline
\end{tabular}


Int.J.Curr.Microbiol.App.Sci (2018) 7(9): 1851-1862

\begin{tabular}{|c|c|c|c|c|c|c|c|c|c|c|c|c|c|}
\hline 23 & $604 \times 444$ & $-12.57 * *$ & $-16.21 * *$ & $-9.97 * *$ & $-14.35 * *$ & $-21.59 * *$ & -3.02 & -1.89 & 0.00 & $-9.20 * *$ & $14.49 * *$ & $-32.66 * *$ & $7.28 *$ \\
\hline 24 & $604 \times 107$ & $-6.53 *$ & $-10.42 * *$ & 0.39 & $-4.42 *$ & -2.15 & 3.46 & 3.21 & 3.21 & $-24.14 * *$ & -4.35 & $-14.82 * *$ & -0.13 \\
\hline 25 & $567 \times 182$ & $7.94 *$ & $-11.55 * *$ & -0.18 & $-4.52 *$ & $-11.04 * *$ & 3.42 & 1.95 & $* 0.64$ & $-13.33 * *$ & $13.04 * *$ & $-24.10 * *$ & $20.40 * *$ \\
\hline 26 & $567 \times 595$ & $-12.81 * *$ & $-14.52 * *$ & $-10.92 * *$ & -1.87 & $-9.17 * *$ & 5.58 & 2.61 & $* 0.64$ & $-5.56 *$ & $23.19 * *$ & $-16.07 * *$ & $33.14 * *$ \\
\hline 27 & $567 \times 562$ & $-11.00 * *$ & $-21.52 * *$ & $-9.42 * *$ & $-13.37 * *$ & $-25.54 * *$ & $-13.44 * *$ & -1.28 & -1.28 & $13.33 * *$ & $47.83 * *$ & $-13.06 * *$ & $37.91 * *$ \\
\hline 28 & $567 \times 347$ & $-10.06 * *$ & $-8.58 * *$ & $-8.78 * *$ & $-12.76 * *$ & -4.52 & $10.99 * *$ & -0.65 & -1.28 & $-5.43 *$ & $26.09 * *$ & $-7.36 * *$ & $46.96 * *$ \\
\hline 29 & $567 \times 444$ & 5.30 & $-10.60 * *$ & -2.10 & $-6.36 * *$ & $-6.39 * *$ & $15.78 * *$ & $-3.77 *$ & -1.92 & $13.33 * *$ & $47.83 * *$ & $11.39 * *$ & $77.46 * *$ \\
\hline 30 & $567 \times 107$ & 1.52 & $-11.37 * *$ & $5.05 *$ & 0.48 & $-18.21 * *$ & -4.93 & -0.65 & -1.92 & $15.56 * *$ & $50.72 * *$ & -0.78 & $57.39 * *$ \\
\hline 31 & $182 \times 595$ & $10.86 * *$ & $8.69 * *$ & $-16.50 * *$ & $-8.03 * *$ & $16.34 * *$ & $28.61 * *$ & 0.65 & -0.64 & $-18.39 * *$ & 2.90 & -3.29 & $36.65 * *$ \\
\hline 32 & $182 \times 562$ & $-15.22 * *$ & $-25.24 * *$ & -2.49 & $-11.58 * *$ & $-16.99 * *$ & $-14.23 * *$ & -1.92 & -1.92 & $19.05 * *$ & $44.93 * *$ & $-6.16 * *$ & $23.87 * *$ \\
\hline 33 & $182 \times 347$ & $-15.06 * *$ & $-13.67 * *$ & 0.32 & $-9.61 * *$ & $-8.93 * *$ & 0.48 & 0.65 & 0.00 & $30.43 * *$ & $73.91 * *$ & $21.94 * *$ & $86.90 * *$ \\
\hline 34 & $182 \times 444$ & 2.76 & $-12.76 * *$ & 1.14 & -3.78 & $-30.05 * *$ & $-13.49 * *$ & -3.14 & -1.28 & 0.00 & $21.74 * *$ & $-26.38 * *$ & $17.28 * *$ \\
\hline 35 & $182 \times 107$ & -3.02 & $-15.33 * *$ & -2.57 & $-7.24 * *$ & 4.20 & $10.18 * *$ & -0.65 & -1.92 & 3.57 & $26.09 * *$ & $11.56 * *$ & $47.25 * *$ \\
\hline 36 & $595 \times 562$ & $-8.56 * *$ & $-10.36 * *$ & $-16.00 * *$ & $-7.47 * *$ & $-6.51 *$ & 3.35 & 0.00 & 0.00 & 3.45 & $30.43 * *$ & -2.81 & $37.34 * *$ \\
\hline 37 & $595 \times 347$ & $-6.71 *$ & -5.18 & $-9.85 * *$ & -0.70 & $-8.30 * *$ & 1.38 & -1.94 & -2.56 & 3.26 & $37.68 * *$ & $-4.82 *$ & $45.90 * *$ \\
\hline 38 & $595 \times 444$ & $-6.34 *$ & $-8.17 * *$ & $-10.47 * *$ & -1.38 & $-5.52 *$ & $16.86 * *$ & -2.52 & -0.64 & 3.45 & $30.43 * *$ & -3.14 & $54.30 * *$ \\
\hline 39 & $595 \times 107$ & $-9.05 * *$ & $-10.83 * *$ & $-9.31 * *$ & -0.10 & $-6.77 *$ & 3.06 & -0.65 & -1.92 & $24.14 * *$ & $56.52 * *$ & $16.24 * *$ & $64.25 * *$ \\
\hline 40 & $562 \times 347$ & $-19.53 * *$ & $-18.21 * *$ & $-5.12 *$ & $-14.29 * *$ & $-23.31 * *$ & $-15.39 * *$ & -1.28 & -1.28 & $7.61 * *$ & $43.48 * *$ & $-20.27 * *$ & $22.21 * *$ \\
\hline 41 & $562 \times 444$ & 5.46 & $-7.00 *$ & -3.50 & $-8.20 * *$ & $-26.46 * *$ & $-9.04 * *$ & -3.14 & -1.28 & $25.00 * *$ & $52.17 * *$ & $-13.18 * *$ & $38.32 * *$ \\
\hline 42 & $562 \times 107$ & 2.20 & $-9.88 * *$ & -1.41 & $-6.14 * *$ & $-5.92 *$ & -0.53 & 0.00 & 0.00 & $10.98 * *$ & $31.88 * *$ & $10.95 * *$ & $30.08 * *$ \\
\hline 43 & $347 \times 444$ & $-6.27 *$ & -4.73 & 0.20 & $-4.68 *$ & $11.08 * *$ & $37.39 * *$ & $-5.66 * *$ & $-3.85 *$ & $-10.87 * *$ & $18.84 * *$ & -0.62 & $58.32 * *$ \\
\hline 44 & $347 \times 107$ & $-25.40 * *$ & $-24.18 * *$ & $-12.43 * *$ & $-16.63 * *$ & 5.00 & $15.85 * *$ & -1.29 & -1.92 & $-21.74 * *$ & 4.35 & $-18.09 * *$ & $25.55 * *$ \\
\hline \multirow[t]{4}{*}{45} & $444 \times 107$ & -0.64 & $-13.26 * *$ & -0.52 & $-5.29 *$ & 1.36 & $25.37 * *$ & $-5.03 * *$ & -3.21 & $-9.52 * *$ & $10.14 * *$ & $-8.17 * *$ & $46.29 * *$ \\
\hline & S.E.D & 0.42 & 0.42 & 0.43 & 0.43 & 0.44 & 0.44 & 0.10 & 0.10 & 0.76 & 0.76 & 10.28 & 10.28 \\
\hline & C.D. 0.05 & 0.85 & 0.85 & 0.86 & 0.86 & 0.89 & 0.89 & 0.20 & 0.20 & 1.52 & 1.52 & 20.71 & 20.71 \\
\hline & C.D. 0.01 & 1.11 & 1.11 & 1.12 & 1.12 & 1.15 & 1.15 & 0.26 & 0.26 & 1.99 & 1.99 & 26.95 & 26.95 \\
\hline
\end{tabular}

$*$, **Significant at $\mathrm{P} \leq 0.05$ and $\mathrm{P} \leq 0.01$ levels probability, respectively. 
Of the 12 characters under study, plant height, number of branches per plant and internodal length largely determine the fruit bearing surface and thus considered as growth attributes. Okra bears pods at almost all nodes on main stem and primary branches. Higher the plant height with more number of branches on the main stem, higher is the number of fruits per plant because of accommodation of more number of nodes for a given internodal length. Shorter distance between nodes accommodates more number of nodes on main stem, which will ultimately lead to higher fruit number and higher fruit production. Hence, positive heterosis is desirable for plant height and number of branches, while negative heterosis is desirable for internodal length to accommodate more number of nodes and to get higher fruit yield in okra. Appreciable amount of the crosses displayed positive standard heterosis for plant height (up to $30.12 \%$ ), no. of branches per plant (up to $26.97 \%$ ), internodal length (up to -19.79\%). Ahmed et al., (1999), Dhankar and Dhankar (2001) and Rewale et al., (2003), Singh et al., (2004), Weerasekara et al., (2007) and Jindal et al., (2009) also reported the similar projections for number of branches in okra. For internodal length, similar projections were also made by Rewale et al., (2003), Singh et al., (2004), and Jindal et al., (2009).

However, days to $50 \%$ flowering and first fruit producing node are the indicators of earliness in okra. Early flowering not only gives early pickings and better returns but also widens fruiting period of the plant. Fruiting at lower nodes is helpful in increasing the number of fruits per plant as well as getting early yields. Negative heterosis is highly desirable for all these three attributes of earliness. In the present study, cross IIHR-562 x IIHR-444 exhibiting high negative heterosis over standard control for days to $50 \%$ flowering $(-8.70 \%)$ out of 45 hybrids, 9 and 9 hybrids showed significant heterosis in desirable direction (negative)over better parent and over standard parent respectively. The cross IIHR-567 x IIHR-107 (Table 2) displaying high negative heterosis over standard control for first fruit producing node $(-9.03 \%)$ among the 45 hybrids developed, 25 hybrids over better parent and 6 hybrids over standard parent showed significantly negative heterosis therefore, it is important to exploit heterosis for earliness in okra. Weerasekara et al., (2007) and Jaiprakashnarayan et al., (2008) also noticed heterosis in desirable direction for days to $50 \%$ flowering in okra. The negative estimates of heterobeltiosis and economic heterosis for earliness revealed the presence of genes for the development of earliness in okra. Mandal and Das (1991), Tippeswamy et al., (2005) and Jindal et al., (2009) also noticed desirable heterosis for first fruit producing node in okra.

Total number of fruits per plant and fruit length, width, and weight are considered to be associated directly with total yield per plant, for which positive heterosis is desirable. The trait fruit length exhibit high magnitude significant heterosis in both the direction of better parent and standard parent. Maximum positive and significant heterosis over better parent $(24.30 \%)$ and over standard parent (13.53\%) was observed in crosses IIHR-478 x IIHR-567. Among 45 hybrids developed, 10 hybrids over better parent and 5 hybrids over standard parent exhibited positive and significant heterosis. The trait fruit diameter exhibit high magnitude significant heterosis in both the better parent and standard parent. The cross IIHR-478 x IIHR-444 exhibited maximum positively significant heterosis over better parent $(16.01 \%)$ and over standard parent (11.90\%). Out of 45 hybrids, 7 and 4 hybrids showed positive and significant heterosis over better parent and over standard parent respectively. The trait average fruit 
weight exhibit high magnitude significant heterosis in both the direction better parent and standard parent. Positively significant heterosis is preferred for this trait. The cross IIHR-604 x IIHR-182, showed maximum positive significant heterosis over better parent $(31.76 \%)$. Whereas, the cross IIHR478 x IIHR-567 showed maximum significant heterosis over standard parent (42.25\%). Among 45 hybrids, 13 hybrids over better parent and 26 hybrids over standard parent exhibited significant positive heterosis (Table 3). Similar results were also reported by Ahmed et al., (1999), Weerasekara et al., (2007) and Jaiprakashnarayan et al., (2008) in okra.

The magnitude of heterosis for number of fruits per plant was significant in both the direction of better parent and where only positive direction was seen in standard parent. Maximum positive significant heterosis was observed in cross IIHR-875 x IIHR-478, 48.91 per cent over better parent and 98.55 per cent over standard parent. Majority of crosses exhibits positive and significant heterosis (Fig. 1). It can be recommended for commercial exploitation.

\section{Acknowledgements}

The authors are highly grateful to the ICARIndian Institute of Horticulture Research Institute, Bengaluru, Karnataka, for providing the germplasm of okra from which the parents for the present study were selected.

\section{References}

Ahmed, N., M.A. Hakim, and M.Y. Gandroo. 1999. Exploitation of hybrid vigour in okra (Abelmoschus esculentus (L.) Moench). Indian Journal of Horticulture. 56:247-251.

Hosamani, R. M., Ajjapalavara, P. S., Basavarajeshwari C., Smith, R. P. and
Ukkand, K. C. 2008. Heterosis for yield and yield components in okra. Karnataka Journal of Agricultural Science. 21(3):473 - 475.

Jaiprakashnarayan, R.P., S.J. Prashanth, R. Mulge, and M.B. Madalageri. 2008. Study on heterosis and combining ability for earliness and yield parameters in okra (Abelmoschus esculentus (L.) Moench). The Asian Journal of Horticulture. 3:136-141.

Jindal, S.K., D. Arora, and T.R. Ghai. 2009. Heterobeltiosis and combining ability for earliness in okra (Abelmoschus esculentus (L.) Moench). Crop Improvement. 36(2):59-66.

Laxmiprasanna, J. R., 1996. Genetic studies in okra (Abelmoschus esculentus (L.) Moench). M.Sc. Thesis, Univ. Agric. Sci., Dharwad.

Mandal, N., and N.D. Das. 1991. Heritability and heterosis study in okra (Abelmoschus esculentus (L.) Moench). Experimental Genetics. 7(1):22-25.

Mehta, N., Asati, B. S. and Mamidwar, S. R. 2007. Heterosis and gene action in okra. Bangladesh Journal of Agricultural Ressearch. 32(3):421 - 432.

Rewale, V.S., V.W. Bendale, S.G. Bhave, R.R. Madav, and B.B. Jadhav. 2003. Heterosis for yield and yield components in okra. Journal of Maharashtra Agricultural Universities. 28:247-249.

Singh, B., S. Singh, A.K. Pal, and M. Rai. 2004. Heterosis for yield and yield components in okra (Abelmoschus esculentus (L.) Moench). Vegetable Science. 31:168-171.

Singh, S. P., Srivastava, J. P. and Singh, H. N., 1975. Heterosis in bhendi (Abelmoschus esculentus (L.) Moench.). South Indian Horticulture. 40:21-27.

Tippeswamy, S., M. Pitchaimuthu, O.P. Dutta, J.S.A. Kumar, and M.C. Ashwini. 2005. Heterosis studies in 
okra (Abelmoschus esculentus (L.) Moench) using male sterile lines. Journal of Asian Horticulture. 2(1):915.

Vijayaraghavan, C., and V.A. Warrier. 1946. Evolution of high yielding hybrid bhindi (Hibiscus esculentus L). 163 p. Proceeding of the 33rd Indian Science Congress, Bangalore, India.
Weerasekara, D., R.C. Jagadeesha, M.C. Wali, P.M. Salimath, R.M. Hosamani, and I.K. Kalappanawar. 2007. Heterosis for yield and yield components in okra. Vegetable Science. 34:106-107.

Wynne, J.C., D.A. Emery, and P.M. Rice. 1970. Combining ability estimates in Arachis hypogeae L. II. Field performance of $\mathrm{F}_{1}$ hybrids. Crop Science. 10:713-715.

\section{How to cite this article:}

Prakash Kerure and Pitchaimuthu, M. 2018. Heterosis Studies in Okra (Abelmoschus esculentus L. Moench). Int.J.Curr.Microbiol.App.Sci. 7(09): 1851-1862.

doi: https://doi.org/10.20546/ijcmas.2018.709.225 